\section{AB1375 RELIABILITY AND VALIDITY OF TURKISH VERSION OF SHORT FORM OF THE SOCIAL ROLE PARTICIPATION QUESTIONNAIRE (S-SRPQ) IN PATIENTS WITH ANKYLOSING SPONDYLITIS}

Y. Akyol ${ }^{1}$, Y. Ulus ${ }^{1}$, Y. Terzi ${ }^{2}$, A. Bilgici ${ }^{1}$, O. Kuru ${ }^{1} .{ }^{1}$ Physical Therapy and Rehabilitation; ${ }^{2}$ Statistics, Ondokuzmayıs university, Samsun, Turkey

Background: Ankylosing spondylitis (AS) is a chronic inflammatory rheumatic disease with a usual onset in the third decade of life, when persons are committed to various social roles that adults fulfil. ${ }^{1}$ When evaluating the outcomes of clinical care, social role participation is an increasingly important outcome, especially when considering diseases with substantial limitations in physical functioning such as inflammatory rheumatic diseases. ${ }^{2}$ The Short Form of the Social Role Participation Questionnaire (s-SRPQ) is a questionnaire which was developed to assess effect of ankylosing spondylitis (AS) on participation. ${ }^{2}$

Objectives: This study aims to evaluate the reliability and validity of the Turkish version of the s-SRPQ in Turkish patients with AS.

Methods: The Turkish version of s-SRPQ questionnaire was obtained after a translation and back translation process. The study sample included $100 \mathrm{AS}$ patients (59 males, 41 females; mean age $42.00 \pm 11.00$ years; range 19 to 69 years). To assess the test-retest reliability of the Turkish s-SRPQ, the questionnaire was reapplied 15 days after the first interview (interclass correlation coefficient, ICC). Cronbach's alpha (a) was used to evaluate the internal consistency. The s-SRPQ was compared with Short Form-36 survey (SF-36), Ankylosing Spondylitis Quality of Life questionnaire (ASQoL), Bath Ankylosing Spondylitis Disease Activity Index (BASDAI), Bath Ankylosing Spondylitis Functional Index (BASFI), and Satisfaction With Life Scale (SWLS) for convergent validity. The internal structure of s-SRPQ was examined by factor analysis.

Results: For s-SRPQ/experienced physical difficulties; the individual item ICC ranged from 0.78 to 1.00 and Cronbach's alpha value ranged from 0.88 to 1.00 . For s-SRPQ/satisfaction with role performance; the individual item ICC ranged from 0.93 to 0.98 and Cronbach's alpha value ranged from 0.96 to 0.99 . KMO values was determined as 0.90 and 0.89 in the s-SRPQ/experienced physical difficulties and s-SRPQ/satisfaction with role performance, respectively. Bartlett's test of sphericity had a $p<0.001$. The Turkish version of $s-S R P Q / e x p e r i e n c e d$ physical difficulties scores negatively correlated with the SWLS and SF-36 sub-parameters scores $(p<0.01)$. There were also strong positive correlations between $s$-SRPQ/ experienced physical difficulties scores and BASDAI, BASFI, and ASQoL scores $(p<0.01)$. The $S R P Q /$ satisfaction with role performance scores positively correlated with the SWLS and SF-36 sub-parameters scores $(p<0.01)$. There were also strong negative correlations between s-SRPQ/experienced physical difficulties scores and BASDAI, BASFI, and ASQoL scores $(p<0.01)$.

Conclusions: Turkish version of s-SRPQ has good comprehensibility, internal consistency, and validity and is an adequate and useful instrument for the assessment of participation in Turkish patients with AS.

\section{REFERENCES:}

[1] Sieper J, Braun J, Rudwaleit M, Boonen A, Zink A (2002) Ankylosing spondylitis: an overview. Ann Rheum Dis 61(Suppl 3):8-18

[2] Oude Voshaar M, van Onna M, van Genderen S, van de Laar M, van der Heijde D, Heuft L, et al. (2016) Development and Validation of a Short Form of the Social Role Participation Questionnaire in Patients with Ankylosing Spondylitis. J Rheumatol 43(7):1386-92

Disclosure of Interest: None declared

DOI: 10.1136/annrheumdis-2018-eular.1198

\section{$\mathrm{AB} 1376$ \\ URINARY MONOCYTE CHEMOATTRACTANT PROTEIN 1 CANNOT DIFFERENTIATE BETWEEN HISTOLOGICAL CLASSES OF LUPUS NEPHRITIS}

R.K. Gone ${ }^{1}$, V. Dhir ${ }^{2}$, A. Sandhu ${ }^{2}$, A. Sharma ${ }^{2}$, M. Rathi ${ }^{3}$, R. Nada ${ }^{4}$, S. Sharma ${ }^{5}$ S.K. Sharma ${ }^{6}{ }^{1}$ Internal medicine, Post graduate institutite of medical education and research, Chandigarh; ${ }^{2}$ Internal medicine; ${ }^{3}$ Department of Nephrology; ${ }^{4}$ Department of Histopathology, ${ }^{5}$ Department of Biochemistry, Post Graduate Institute of Medical Education and Research; ${ }^{6}$ Internal medicine, Post Graduate Medical Education Education and Research, Chandigarh, India

Background: Lupus Nephritis, one of the commonest manifestation of SLE, seen in $60 \%$ of adult SLE patients at some point of time, carries poor prognosis when compared to those with no renal involvement. The natural course of the LN has recurrent flares, necessitating the need for early detection and treatment. Despite being the gold standard in diagnosing Lupus nephritis and its severity, renal biopsy is an invasive procedure with potential complications, and difficult to repeat. Hence, a novel biomarker, reflecting the disease activity and severity is needed to predict flare. Monocyte chemoattractant Protein 1, a chemokine produced locally during Lupus Nephritis flare, was shown in many previous studies as a promising biomarker

Objectives: We studied the role of Urinary MCP-1 as a biomarker of disease activity in LN and compare its value among different histological classes of Lupus nephritis

Methods: This is a case-control study conducted at a tertiary care centre in North India from July 2016 to December 2017. Cases were those patients undergoing renal biopsy satisfying the inclusion criteria set for SLE with $\operatorname{LN}(n=36)$. Controls were patients of SLE without active LN [Control I-prior LN $(n=11)$ and Control IInever had LN $(n=15)]$. Urinary MCP-1 measurement was done using Sandwich ELISA kit

Results: The mean age in cases was $31.1 \pm 10.2$ years and mean age in controlwas $34.6 \pm 8.0$ and in control II was $36.3 \pm 10.4$. Urinary MCP-1 values in cases $(1214 \pm 1467.1 \mathrm{pg} / \mathrm{mg})$ was significantly higher compared to Controls $(184.5$ $\pm 186.8)$. However, no significant difference was observed between Control I (170.5 \pm 150.8$)$ and Control II (194 \pm 214$)$. Urinary MCP-1 levels show significant correlation when compared with classical disease markers like 24 hour proteinuria, 24 hour PCR, Spot PCR and SLEDAl. Cut off value $(339 \mathrm{pg} / \mathrm{mg}, \mathrm{p}<0.001)$ obtained from ROC curve has sensitivity and specificity of $80 \%$ and $92 \%$ respectively. However, there was no significant difference of Urinary MCP-1 levels was observed among different classes of $L N(p=0.593)$. ROC curve comparison of urinary MCP-1 $(A \cup C=0.879)$ with 24 hour urine protein to creatinine ratio ( $A \cup C=0.964)$ and spot urine protein to creatinine ratio $(A \cup C=0.872)$ showed that it is not a better marker of disease activity than both of them. Urinary MCP-1 levels didn't show significant correlation with Renal activity index calculated on renal biopsy.

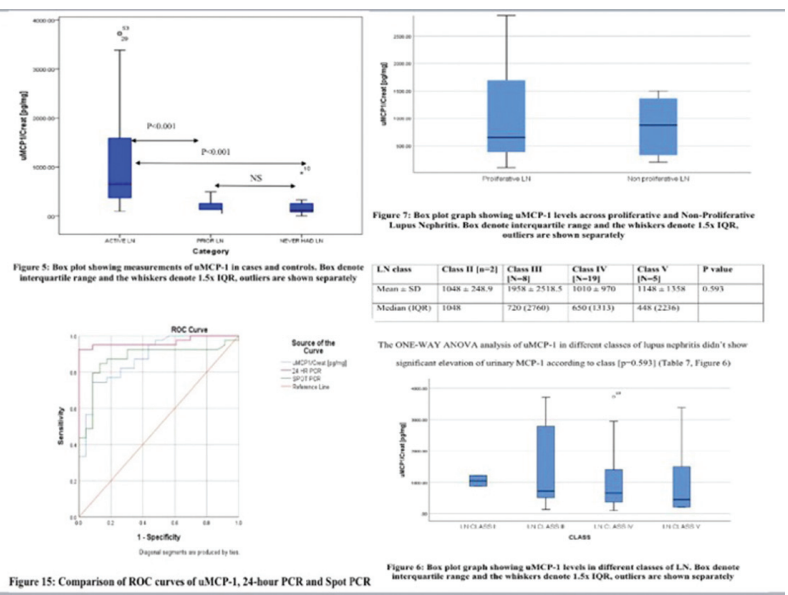

Conclusions: In this study we found higher Urinary MCP-1 levels in active LN compared to Inactive LN and its level was correlated well with the presently used disease markers. However there was no significant difference among different histological classes of LN

Disclosure of Interest: None declared

DOI: 10.1136/annrheumdis-2018-eular.6733

\section{AB1377 15 THE PATIENT GLOBAL HEALTH ASSESSMENT RELIABLE IN JUVENILE IDIOPATHIC ARTHRITIS (JIA)?}

R. Trachtman, C.M. Wang, J. Szymonifka, K.B. Onel. Hospital for Special Surgery/ Weill Cornell Medicine, New York, USA

Background: JIA is a chronic autoimmune disease that poses many challenges. There is increasing recognition of the importance of patient-reported outcomes (PROs) and newer PROs are being developed and more widely utilised both in clinical care and in research, However, their performance and reliability remain unclear

Objectives: This study seeks to evaluate: ${ }^{1}$ performance of the patient global health assessment (PGA) compared to standard disease activity measures in children with $\mathrm{JIA},{ }^{2}$ correlations of the PGA with socioeconomic status (SES) in JIA and $^{3}$ relationship between PGA and physician global health assessment in JIA.

Methods: A convenience sample of patients with JIA $(n=47)$ aged $2-18$ were recruited from a single centre. Patients aged $\geq 10$ years completed the questionnaire, and parents of patients aged 2-9 completed a proxy questionnaire for their child. Correlations between ${ }^{1}$ the PGA and disease activity, as measured by the Juvenile Disease Activity Score-71 (JADAS-71), ${ }^{2}$ the PGA and physician global health assessment, and ${ }^{3}$ the physician global health assessment and the JADAS 71 were evaluated using Spearman correlation coefficients. PGAs were compared by age, sex, insurance status, race, and ethnicity; and differences between 